\title{
Quantum Theory of Irreversibility
}

\author{
A. Prez-Madrid ${ }^{1}$ \\ Departament de Fsica Fonamental. Facultat de Fsica. Universitat de Barcelona. \\ Diagonal 647, 08028 Barcelona. Spain
}

\begin{abstract}
A generalization of the Gibbs-von Neumann relative entropy is proposed based on the quantum BBGKY [Bogolyubov-Born-Green-Kirkwood-Yvon] hierarchy as the nonequilibrium entropy for an N-body system. By using a generalization of the Liouville-von Neumann equation describing the evolution of a density superoperator, it is demonstrated that the entropy production for an isolated system is non-negative, which provides an arrow of time. Moreover, following the procedure of non-equilibrium thermodynamics a master matrix is introduced for which a microscopic expression is obtained. Then, the quantum Boltzmann equation is derived in terms of a transition superoperator related to that master matrix.
\end{abstract}

Key words: quantum statistical mechanics, nonequilibrium and irreversible thermodynamics, kinetic and transport theory of gases

\section{Introduction}

According to the mechanicistic interpretation of the physical world, the basic laws of nature are deterministic and time reversible. However, at the macroscopic level we observe irreversible processes related to energy degradation which generate entropy. How do we reconcile the 'spontaneous production of entropy' with the time reversibility of the microscopic equations of motion?. This is a problem not solved in a clear and definitive way yet, thus it still constitutes an open problem. Attempts to address this have been made based on coarse-graining procedures [1]-[3]. In a previous work [4] we showed that in the framework of the classical BBGKY- hierarchy which explicitly incorporates the correlation between all the particle clusters in the system, macroscopic irreversibility arises without coarse-grainig procedures. All that is needed is a coherent definition of the entropy given through a generalization of the Gibbs

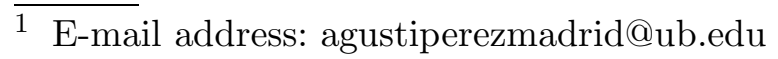


relative entropy in terms of the distribution vector in addition to the generalized Liouville equation. Based on the fact that the generalized Liouville equation introduces a trace preserving transformation in the set of distribution vectors, we obtained a non-negative entropy production for the isolated system which manifests the existence of a classical time arrow by appealing to the underlying microscopic dynamics only.

Here we develop the quantum version of this previous analysis. The quantum counterpart of the distribution vector is the density superoperator evolving according to the generalized Liouville-von Neumann equation. Moreover, we propose a generalization of the Gibbs-von Neumann relative entropy in terms of the density superoperator, in the spirit of the generalized Gibbs relative entropy postulate. As in the classical approach, here, the generalized Liouvillevon Neumann equation introduces a trace preserving transformation aswell. From this we obtain a non-negative entropy production for the isolated system, providing an arrow of time and giving a microscopic basis to the second law of Thermodynamics. After obtaining that, through a thermodynamic analysis, the quantum Boltzmann equation follows in a natural way.

We have structured the paper as follows. In section 2, we introduce the Hamiltonian dynamic of the N-body system obtaining the generalized Liouville-von Neumann equation. In section 3, we compute the entropy production. Section 4 is devoted to the non-Equilibrium Thermodynamic analysis of the system obtaining the quantum Boltzmann equation. Finally in section 5, we emphasize our main conclusions.

\section{Hamiltonian Dynamics}

Let's consider a dynamical system of $\mathrm{N}$ identical particles whose Hamiltonian is given by the $\mathrm{N}$-particle Hamiltonian

$$
H^{(N)}=\sum_{j=1}^{N} H(j)+\frac{1}{2} \sum_{j \neq k=1}^{N} \phi(j, k),
$$

where $H(j)$ denotes the individual energy of the $j$-th particle and $\phi(j, k)$ the interaction energy between the $j$-th and $k$-th particles. The state of the system is completely specified at a given time by the N-particle density operator $D^{(N)}(1, \ldots, N)$ which evolves according to the Liouville-von Neumann equation $[5]$

$$
i \hbar \frac{\partial}{\partial t} D^{(N)}=\left[H^{(N)}, D^{(N)}\right]
$$


In the Liouville representation, where the density operator is represented as a vector in the Liouville space [7]-[10], the Liouville-von Neumann equation becomes

$$
i \hbar \frac{\partial}{\partial t} D_{\alpha \beta}^{(N)}=L_{\alpha \beta, \gamma \delta} D_{\gamma \delta}^{(N)}
$$

where repeated Greek indices mean summation and the Liouvillian $L$ is defined through

$$
L_{\alpha \beta, \gamma \delta}=\left(H_{\alpha \delta}^{(N)} \delta_{\beta \gamma}-H_{\gamma \beta}^{(N)} \delta_{\alpha \delta}\right)
$$

However, a strictly equivalent alternative description of the state of the system can be given in terms of the set of n-particle reduced density operators [5], [6]

$$
\mathcal{D} \equiv\left\{D^{(0)}, D^{(1)}, \ldots \ldots, D^{(N)}\right\}
$$

where $\mathcal{D}$ constitutes a density superoperator whose components are the nparticle reduced density operators $D^{(n)}(1, \ldots, n)$ defined through

$$
D^{(n)}(1, \ldots, n)=\frac{N !}{(N-n) !} \operatorname{Tr}_{(n+1, \ldots, N)} D^{(N)}(1, \ldots, N)
$$

and $D_{\alpha \beta}^{(0)}=\delta_{\alpha \beta}$ (i.e. $D^{(0)}$ coincides with the unit matrix). These operators satisfy

$$
\operatorname{Tr}_{(1, \ldots, n)}\left\{D^{(n)}(1, \ldots, n)\right\}=\frac{N !}{(N-n) !}
$$

The superoperator $\mathcal{D}$ can be represented alternatively as a $(N+1) \times(N+1)$ block diagonal matrix in an abstract space

$$
\mathcal{D}=\sum_{n=0}^{N} \frac{1}{n !} \mathcal{I}_{n} \otimes D^{(n)},
$$

where the $(N+1) \times(N+1)$ matrices $\mathcal{I}_{n}$ are characterized for having zero everywhere except for the n-th row and the n-th column and satisfying

$$
\mathcal{I}_{n} \odot \mathcal{I}_{p}=\delta_{n p}
$$

which defines the inner product in this abstract space. Moreover, in view of Eq. (7)

$$
\operatorname{Tr}\{\mathcal{D}\}=\sum_{n=0}^{N} \frac{1}{n !} \operatorname{Tr}_{(1, \ldots, n)}\left\{D^{(n)}\right\}=2^{N}
$$

On the other hand, the dynamics of the $D^{(n)}$ follows after performing the operation $\underset{(n+1, \ldots, N)}{\operatorname{Tr}}$ on both sides of Eq. (2). Thus, we obtain the so-called quantum BBGKY-hierarchy of equations which can be written in a compact 
way as a generalized Liouville equation

$$
i \hbar \frac{\partial}{\partial t} \mathcal{D}(t)=\mathcal{L} \odot \mathcal{D}(t)
$$

At this point, it is convenient to introduce the projection operators $\mathcal{P}$ and $\mathcal{Q}$, its complement with respect to the identity (i.e., $\mathcal{P} \mathcal{L}+\mathcal{Q} \mathcal{L}=\mathcal{L}$ ), which give the diagonal and non-diagonal part of the generalized Liouvillian $\mathcal{L}$, respectively. This projection operators are defined according to

$$
\mathcal{P} \mathcal{L}=\sum_{n=0}^{N} \sum_{p=0}^{N} \delta_{p n} \mathcal{I}_{n} \otimes \mathcal{I}_{n} \otimes L^{(n)},
$$

where $L^{(n)}$ is the n-particle Liouvillian

$$
L_{\alpha \beta, \gamma \delta}^{(n)}=\left(H_{\alpha \delta}^{(n)} \delta_{\beta \gamma}-H_{\gamma \beta}^{(n)} \delta_{\alpha \delta}\right)
$$

and $H^{(n)}$ is the n-particle Hamiltonian. Thus, it becomes

$$
\mathcal{P} \mathcal{L} \odot \mathcal{D}=\sum_{n=0}^{N} \frac{1}{n !} \mathcal{I}_{n} \otimes\left(L^{(n)} \odot D^{(n)}\right) .
$$

In addition, when the inner product $\odot$ involves two densities operators $U$ and $V$, it should be understood as

$$
A \odot B=A_{\alpha \beta} B_{\beta \alpha}
$$

On the other hand,

$$
\mathcal{Q} \mathcal{L}=\sum_{n=0}^{N} \mathcal{I}_{n} \otimes \sum_{p=0}^{N} \delta_{p(n+1)} \operatorname{Tr}_{p}\left\{\mathcal{I}_{p} \otimes \Delta^{(p)}\right\},
$$

where we have defined

$$
\Delta_{\alpha \beta, \gamma \delta}^{(p)}=\left(F_{\alpha \delta}^{(p)} \delta_{\beta \gamma}-F_{\gamma \beta}^{(p)} \delta_{\alpha \delta}\right)
$$

and

$$
F^{(p)}(1, \ldots, p)=\sum_{j=1}^{p-1} \phi(j, p)
$$

Therefore,

$$
\mathcal{Q L} \odot \mathcal{D}=\sum_{n=0}^{N} \frac{1}{n !} \mathcal{I}_{n} \otimes \operatorname{Tr}_{(n+1)}\left\{\Delta^{(n+1)} \odot D^{(n+1)}\right\} .
$$

Hence, Eq. (11) can be rewritten as

$$
i \hbar \frac{\partial}{\partial t} \mathcal{D}(t)-\mathcal{P} \mathcal{L} \odot \mathcal{D}=\mathcal{Q L} \odot \mathcal{D} .
$$


According to Eqs. (12)-(18), one of the components of Eq. (20) will read

$$
\begin{aligned}
& i \hbar \frac{\partial}{\partial t} D_{\alpha \beta}^{(n)}-\left(H_{\alpha \delta}^{(n)} D_{\beta \delta}^{(n)}-H_{\gamma \beta}^{(n)} D_{\alpha \gamma}^{(n)}\right) \\
& =\operatorname{Tr}_{(n+1)}\left\{F_{\alpha \delta}^{(n+1)} D_{\beta \delta}^{(n+1)}-F_{\gamma \beta}^{(n+1)} D_{\alpha \gamma}^{(n+1)}\right\} .
\end{aligned}
$$

Without the right hand side term in this equation, the evolution of $D^{(n)}$ would be unitary. In the next section we will show that irreversibility is manifested in the dynamics of the system when the adequate description, i.e. in terms of the set $\mathcal{D}$ of all the n-particle reduced density operators is used.

\section{Non-equilibrium entropy and irreversibility}

As the expression for the non-equilibrium entropy we propose

$$
\begin{aligned}
S & =k_{B} \operatorname{Tr}\left\{\mathcal{D}\left(\ln \mathcal{D}_{o}-\ln \mathcal{D}\right)\right\}+S_{o} \\
& =k_{B} \sum_{n=0}^{N} \frac{1}{n !} \mathcal{I}_{n} \otimes D^{(n)} \odot \sum_{p=0}^{N} \mathcal{I}_{p} \otimes\left(\ln D_{o}^{(p)}-\ln D^{(p)}\right)+S_{o} \\
& =k_{B} \sum_{n=0}^{N} \frac{1}{n !} D^{(n)} \odot\left(\ln D_{o}^{(n)}-\ln D^{(n)}\right)+S_{o},
\end{aligned}
$$

a functional of $D^{(n)}$, generalizing the Gibbs-von Neumann entropy postulate [11], [12], based on the fact that the superoperator $\mathcal{D}$ determines the state of the system. This entropy is analogue to the relative or Kullback entropy [17], [18]. Here, $k_{B}$ is the Boltzmann constant, $S_{o}$ the equilibrium entropy and $\mathcal{D}_{o}$ is assumed to be the equilibrium value of $\mathcal{D}$ which corresponds with $S_{o}$, satisfying $\mathcal{L D}_{o}=0$. It is known that $S$ is a concave functional of $\mathcal{D}$, and will never decrease under a linear stochastic transformation like the one induced by Eq. (11) [14]-[18]

$$
\mathcal{D}(t+\tau)=(\mathcal{U}+\tau \mathcal{L}) \odot \mathcal{D}(t)
$$

with $\mathcal{U}$ being the unit superoperator

$$
\mathcal{U}=\sum_{n=0}^{N} \mathcal{I}_{n} \otimes \mathcal{I}_{n} \otimes U^{(n \mid n)}
$$

and

$$
U^{(n \mid n)}=\sum_{\alpha \beta, \gamma \delta} I_{\alpha \beta, \gamma \delta}|\alpha\rangle\langle\beta|\otimes| \gamma\rangle\langle\delta|,
$$

where $I_{\alpha \beta, \gamma \delta}=\delta_{\gamma \beta} \delta_{\alpha \delta}$ is the unit tetradic [19]. Therefore, the entropy we propose is coherent with the second law according to which $S$ increases in 
irreversible processes such as the relaxation to equilibrium from an initially non-equilibrium state. To prove this we compute the rate of change of $S$ which can be obtained by differentiating Eq. (22) with the help of Eq. (11)

$$
\begin{aligned}
\frac{d S}{d t} & =k_{B} \operatorname{Tr}\left\{\frac{\partial \mathcal{D}}{\partial t}\left(\ln \mathcal{D}_{o}-\ln \mathcal{D}\right)\right\} \\
& =\sigma_{1}+\sigma_{2} \geq 0
\end{aligned}
$$

where

and

$$
\sigma_{1}=-i \hbar^{-1} k_{B} \operatorname{Tr}\left\{\mathcal{P} \mathcal{L} \odot \mathcal{D}\left(\ln \mathcal{D}_{o}-\ln \mathcal{D}\right)\right\}
$$

$$
\sigma_{2}=-i \hbar^{-1} k_{B} \operatorname{Tr}\left\{\mathcal{Q} \mathcal{L} \odot \mathcal{D}\left(\ln \mathcal{D}_{o}-\ln \mathcal{D}\right)\right\} .
$$

Eq. (26) constitutes the entropy production corresponding to the relaxation passing from a non-equilibrium state to equilibrium. By using Eqs. (12)-(19) in addition to the cyclic invariance property of the trace, one has

$$
\begin{aligned}
\sigma_{1} & =-i \hbar^{-1} k_{B} \sum_{n=0}^{N} \frac{1}{n !} L^{(n)} \odot D^{(n)} \odot\left(\ln D_{o}^{(n)}-\ln D^{(n)}\right) \\
& =-i \hbar^{-1} k_{B} \sum_{n=0}^{N} \frac{1}{n !}\left[H^{(n)}, D^{(n)}\right] \odot\left(\ln D_{o}^{(n)}-\ln D^{(n)}\right) \\
& =i \hbar^{-1} k_{B} \sum_{n=0}^{N} \frac{1}{n !}\left\{H^{(n)} \odot\left[\ln D_{o}^{(n)}, D^{(n)}\right]\right\} \\
& =i \hbar^{-1} k_{B} \sum_{n=0}^{N} \frac{1}{n !}\left[H^{(n)}, \ln D_{o}^{(n)}\right] \odot D^{(n)}
\end{aligned}
$$

and

$$
\begin{aligned}
\sigma_{2} & =-i \hbar^{-1} k_{B} \sum_{n=0}^{N} \frac{1}{n !} \operatorname{Tr}_{(n+1)}\left\{\Delta^{(n+1)} \odot D^{(n+1)}\right\} \odot\left(\ln D_{o}^{(n)}-\ln D^{(n)}\right) \\
& =-i \hbar^{-1} k_{B} \sum_{n=0}^{N} \frac{1}{n !} \operatorname{Tr}_{(n+1)}\left\{\left[F^{(n+1)}, D^{(n+1)}\right]\right\} \odot\left(\ln D_{o}^{(n)}-\ln D^{(n)}\right) \\
& =i \hbar^{-1} k_{B} \sum_{n=0}^{N} \frac{1}{n !} \operatorname{Tr}_{(n+1)}\left\{F^{(n+1)} \odot\left[\left(\ln D_{o}^{(n)}-\ln D^{(n)}\right), D^{(n+1)}\right]\right\} .
\end{aligned}
$$

These are zero at equilibrium when $D^{(n)}=D_{o}^{(n)}$, but in any other case these are not necessarily zero because neither the density $D^{(n+1)}$ commutes with $D^{(n)}$ and $D_{o}^{(n)}$ nor $D_{o}^{(n)}$ commutes with $H^{(n)}$, in general. Hence, according to our previous discussion related to the concave character of the entropy $S$ and its consequences, $\sigma$ should be non negative. This provides a time arrow inherent to the irreversible macroscopic manifestation of the dynamics of the system.

As known due to the unitary character of the time-evolution operator related to the Liouville-von Neumann equation giving the dynamics of the N-particle 
density operator, the von Neumann entropy is a constant of motion. Nevertheless, as we have seen here, this is not the case for the generalized Gibbs-von Neumann entropy, based on the fact that the dynamics of the density superoperator given through the genaralized Liouville-von Neumann equation is not unitary. In addition, as compared to other definitions of entropy, Eq. (22) does not contain any coarse-grainig. In the light of these facts not contained in

other definitions of the entropy in the literature the strength of our definition (22) resides.

\section{Non-equilibrium thermodynamics analysis}

Here we proceed as in Ref. [20], which constitutes the standard way of Nonequilibrium thermodynamics. Thus, for small deviations from equilibrium we assume that $\mathcal{D}$ and $\mathcal{D}_{o}$ are diagonal in a common basis. According to this, the entropy (22) becomes up to second order

$$
S=-\frac{1}{2} k_{B} \operatorname{Tr}\left\{\delta \mathcal{D} \mathcal{D}_{o}^{-1} \delta \mathcal{D}\right\}+S_{o}
$$

where $\delta \mathcal{D}=\mathcal{D}-\mathcal{D}_{o}$. Therefore, the entropy production is

$$
\begin{aligned}
\sigma & =\frac{d S}{d t}=-\frac{1}{2} k_{B} \operatorname{Tr}\left\{\frac{\partial \mathcal{D}}{\partial t}\left(\mathcal{D}_{o}^{-1} \delta \mathcal{D}+\delta \mathcal{D D}_{o}^{-1}\right)\right\} \\
& =\operatorname{Tr}\left\{\frac{\partial \mathcal{D}}{\partial t} \mathcal{X}\right\}
\end{aligned}
$$

where the thermodynamic force

$$
\mathcal{X} \equiv \delta S / \delta \mathcal{D}=-\frac{1}{2} k_{B}\left(\mathcal{D}_{o}^{-1} \delta \mathcal{D}+\delta \mathcal{D} \mathcal{D}_{o}^{-1}\right)
$$

is conjugated to the flux $\partial \mathcal{D} / \partial t$. From Eq. (32) the phenomenological relation

$$
\frac{\partial \mathcal{D}}{\partial t}=\mathcal{R} \odot \mathcal{X}
$$

is inferred, where

$$
\mathcal{R}=\sum_{n=0}^{N} \sum_{p=0}^{N} \mathcal{I}_{n} \otimes \mathcal{I}_{p} \otimes R^{(n \mid p)}
$$

is a phenomenological supermatrix which due to the Hermitian character of the force $\mathcal{X}$, we choose Hermitian on the right-hand side entry. This implies that

$$
R_{\alpha \beta, \gamma \delta}^{(n \mid p)}=R_{\alpha \beta, \delta \gamma}^{*(n \mid p)}
$$


where the superscript $*$ stands for the complex conjugate. Using this property, one can replace $\mathcal{X}$ in Eq. (34) by

$$
\mathcal{X}=-k_{B} \mathcal{D}_{o}^{-1} \delta \mathcal{D}=\sum_{n=0}^{N} \frac{1}{n !} \mathcal{I}_{n} \otimes X^{(n)},
$$

a nonHermitian form. Furthermore, due to the fact that $\mathcal{D}$ is normalized, from Eq. (34) we infer that $\operatorname{Tr}(\mathcal{R} \odot \mathcal{X})=0$, which leads to the following constraints

$$
\sum_{\alpha} R_{\alpha \alpha, \gamma \delta}^{(n \mid p)}=0
$$

By substituting the phenomenological relation (34) into Eq. (32) we reach the expression

$$
i \hbar^{-1} \sum_{n=0}^{N} \frac{1}{n !} \operatorname{Tr}_{(n+1)}\left\{\left[X^{(n)}, D^{(n+1)}\right] \odot F^{(n+1)}\right\}=\sum_{n=0}^{N} \sum_{p=0}^{N} X^{(n)} \odot R^{(n \mid p)} \odot X^{(p)}
$$

a kind of Green-Kubo relation, also obtained by using Eqs. (29) and (30) in the near-equilibrium approximation.

Additionally, $\mathcal{R}$ should be Hermitian as predicted by the Onsager symmetry relations, which reflects in

$$
R_{\alpha \beta, \gamma \delta}^{(n \mid p)}=R_{\delta \gamma, \beta \alpha}^{*(p \mid n)}
$$

This leads to the constraint

$$
\sum_{\alpha} R_{\gamma \delta, \alpha \alpha}^{(n \mid p)}=0
$$

and to the hermiticity in the left-hand side entry

$$
R_{\alpha \beta, \gamma \delta}^{(n \mid p)}=R_{\beta \alpha, \gamma \delta}^{*(n \mid p)}
$$

With Eq. (37), Eq. (34) may be written in the form

$$
\frac{\partial \mathcal{D}}{\partial t}=-\mathcal{M} \odot \delta \mathcal{D}
$$

where the master matrix is defined by

$$
\mathcal{M} \equiv-k_{B} \mathcal{R} \mathcal{D}_{o}^{-1}
$$

According to the Onsager relations, it follows that the master matrix satisfies

$$
\mathcal{M D}_{o}=-k_{B} \mathcal{R}=-k_{B} \mathcal{R}^{\dagger}=\mathcal{D}_{o} \mathcal{M}^{\dagger}
$$


where $\nmid$ refers to the Hermitian conjugate. This results in the detailed balance for transition probabilities, and vice versa, when those are introduced.

By using the constraint Eq. (41) one has

$$
\mathcal{M} \odot \mathcal{D}_{o}=-k_{B} \mathcal{R} \mathcal{D}_{o}^{-1} \odot \mathcal{D}_{o}=-k_{B} \sum_{n=0}^{N} \sum_{p=0}^{N} \mathcal{I}_{n} \otimes R^{(n \mid p)} \odot D^{(0)}=0
$$

which proves that the equilibrium density superoperator is a right eigenfunction of the master supermatrix with an eigenvalue zero. Here, we have used the fact that

$$
\mathcal{I}_{n} \mathcal{I}_{p}=\mathcal{I}_{n} \delta_{n p}
$$

So that, Eq. (43) can be rewritten as

$$
\frac{\partial \mathcal{D}}{\partial t}=-\mathcal{M} \odot \mathcal{D}
$$

Now, by substitution of the master equation (48) into the generalized Liouville equation (20) we obtain

$$
\frac{\partial}{\partial t} \mathcal{D}(t)+i \hbar^{-1} \mathcal{P} \mathcal{L} \odot \mathcal{D}(t)=-\mathcal{Q} \mathcal{M} \odot \mathcal{D}(t)
$$

where $\mathcal{Q} \mathcal{M}$ should have the same structure as $\mathcal{Q} \mathcal{L}$, thus

$$
\mathcal{Q} \mathcal{M}=\sum_{n, p=0}^{N} p ! \delta_{p(n+1)} \mathcal{I}_{n} \otimes \operatorname{Tr}_{(n+1)} \quad\left\{\mathcal{I}_{p} \otimes M^{(n+1 \mid p)}\right\}
$$

and

$$
\mathcal{Q} \mathcal{M} \odot \mathcal{D}=\sum_{n, p=0}^{N} \delta_{p(n+1)} \mathcal{I}_{n} \otimes \operatorname{Tr}_{(n+1)}\left\{M^{(n+1 \mid p)} \odot D^{(p)}\right\} .
$$

This last equation allows us to write

$$
\begin{gathered}
\frac{\partial}{\partial t} D_{\alpha \beta}^{(n)}+i \hbar^{-1}\left(H_{\alpha \delta}^{(n)} D_{\beta \delta}^{(n)}-H_{\gamma \beta}^{(n)} D_{\alpha \gamma}^{(n)}\right)= \\
-\sum_{p} \delta_{p(n+1)} \operatorname{Tr}_{(n+1)}\left(M_{\alpha \beta, \gamma \delta}^{(n+1 \mid p)} D_{\delta \gamma}^{(p)}\right)
\end{gathered}
$$

equivalent to Eq. (21).

Here, Eq. (52) can be put into a more common form by introducing the transition superoperator $W_{\alpha \beta, \gamma \delta}^{(n \mid p)}$ defined through

$$
W_{\alpha \beta, \gamma \delta}^{(n \mid p)}=-M_{\gamma \delta, \alpha \beta}^{(p \mid n)}+\Lambda_{\alpha \beta, \gamma \delta}^{(n \mid p)},
$$

where

$$
\Lambda_{\alpha \beta, \gamma \delta}^{(n \mid p)}=I_{\alpha \beta, \gamma \delta} \Psi_{\gamma \delta}^{(n)} \delta_{n p}
$$


The auxiliary matrix $\Psi_{\gamma \delta}^{(n)}$ is not arbitrary, satisfying

$$
\sum_{\alpha} W_{\alpha \alpha, \gamma \delta}^{(n \mid p)}=\delta_{\gamma \delta} \Psi_{\gamma \gamma}^{(n)} \delta_{n p}
$$

in view of Eq. (38). This means that $\sum_{\alpha} W_{\alpha \alpha, \gamma \delta}^{(n \mid p)}$ is a diagonal matrix. In order to satisfy detailed balance, $\Lambda_{\alpha \beta, \gamma \delta}^{(n \mid p)}$ should be Hermitian. Hence, with Eq. (45) one has

$$
D_{o, \delta \gamma}^{(p)} W_{\gamma \delta, \alpha \beta}^{(p \mid n)}=W_{\beta \alpha, \delta \gamma}^{(n \mid p)} D_{o, \gamma \delta}^{(p)}
$$

constituting the expression of the detailed balance principle. Thus, projecting Eq. (52) onto its diagonal part by means of the tetradic operator $P_{i j, k l}=$ $\delta_{i j} \delta_{i k} \delta_{j l}[19]$

$$
\begin{gathered}
P \odot\left(\frac{\partial}{\partial t} D^{(n)}+i \hbar^{-1}\left[H^{(n)}, D^{(n)}\right]\right)= \\
P \odot\left(-\sum_{p} \delta_{p(n+1)} \operatorname{Tr}_{(n+1)}\left(M^{(n+1 \mid p)} \odot D^{(p)}\right)\right),
\end{gathered}
$$

and by using Eqs. (53)-(55) we obtain

$$
\begin{gathered}
\frac{\partial}{\partial t} D_{\alpha \alpha}^{(n)}+i \hbar^{-1}\left(H_{\alpha \gamma}^{(n)} D_{\gamma \alpha}^{(n)}-H_{\alpha \gamma}^{(n)} D_{\gamma \alpha}^{(n)}\right)= \\
\operatorname{Tr}_{(n+1)}\left\{D_{\gamma \delta}^{(n+1)} W_{\gamma \delta, \alpha \alpha}^{(n+1 \mid n+1)}-\left(\sum_{\gamma} W_{\gamma \gamma, \alpha \alpha}^{(n+1 \mid n+1)}\right) D_{\alpha \alpha}^{(n+1)}\right\} .
\end{gathered}
$$

For $n=1$

$$
\begin{aligned}
& \frac{\partial}{\partial t} D_{\alpha \alpha}^{(1)}+i \hbar^{-1}\left(H_{\alpha \gamma}^{(1)} D_{\gamma \alpha}^{(1)}-H_{\alpha \gamma}^{(1)} D_{\gamma \alpha}^{(1)}\right)= \\
& \operatorname{Tr}_{(2)}\left\{D_{\gamma \delta}^{(2)} W_{\gamma \delta, \alpha \alpha}^{(2 \mid 2)}-\left(\sum_{\gamma} W_{\gamma \gamma, \alpha \alpha}^{(2 \mid 2)}\right) D_{\alpha \alpha}^{(2)}\right\} .
\end{aligned}
$$

By neglecting the correlations which means assuming $D_{\alpha \beta}^{(2)}(12)=D_{\alpha \beta}^{(1)}(1) D_{\alpha \beta}^{(1)}(2)$, we obtain

$$
\begin{aligned}
& \frac{\partial}{\partial t} D_{\alpha \alpha}^{(1)}(1)+i \hbar^{-1}\left(H_{\alpha \gamma}^{(1)} D_{\gamma \alpha}^{(1)}(1)-H_{\alpha \gamma}^{(1)} D_{\gamma \alpha}^{(1)}(1)\right) \\
& =\operatorname{Tr}_{(2)}\left\{D_{\gamma \delta}^{(1)}(1) D_{\gamma \delta}^{(1)}(2) W_{\gamma \delta, \alpha \alpha}^{(2 \mid 2)}(12)-\left(\sum_{\gamma} W_{\gamma \gamma, \alpha \alpha}^{(2 \mid 2)}(12)\right) D_{\alpha \alpha}^{(1)}(1) D_{\alpha \alpha}^{(1)}(2)\right\}
\end{aligned}
$$

which constitutes the quantum Boltzmann equation. 


\section{Conclusions}

Here we have shown a representation of the statistical description of an N-body system in terms of a density superoperator which reveals the irreversibility at the macroscopic level. In the scenario drawn by the Liouville-von Neumann equation with its associated Gibbs-von Neumann relative entropy, irreversibility is hidden because of the inherent unitary evolution of the N-particle density operator. Nonetheless, in the framework of the quantum BBGKY-hierarchy the density superoperator follows a non-unitary dynamics given by the generalized Liouville-von Neumann equation. Thus, the generalization of the Gibbsvon Neumann entropy in terms of the density supoeroperator we propose is not conserved by this dynamics. Moreover, this dynamics is trace preserving which as is known [14]-[18], ensures the non-negative character of the entropy production which is coherent with the second law of Thermodynamic.

In addition, following the procedure of the non-Equilibrium Thermodynamics we introduce a master matrix which satisfies a kind of Green-Kubo relation. This, allows us to transform the BBGKY-hierarchy into a hierarchy of master equations which we rewrite in terms of a transition superoperator related to the master matrix. Once this has been done, by neglecting correlations in the spirit of the Boltzmann's Stossahlansatz, we derive the quantum Boltzmann equation.

To summarize, our point is that the framework of the BBGKY-hierarchy which explicitly incorporates the correlations between all the particle clusters in the system constitutes the most appropriate description leading to irreversibility at the macroscopic level. To manifest this irreversibility all that is needed is a coherent definition of the entropy which is our generalaized Gibbs-von Neumann relative entropy in addition to the generalized Liouville-von Neumann equation.

In this way a macroscopic time arrow arises in a natural way in the system. Hence, we can say that our analysis constitutes a microscopic basis of the macroscopic irreversibility.

\section{References}

[1] J. R. Dorfman,P. Gaspard,and T. Gilbert, Phys. Rev. E 66, (2002) 026110-1 026110-9.

[2] J. Vollmer, T. Tél and W. Breymann, Phys. Rev. Lett. 79, (1997) 2759-2762.

[3] L. Rondoni and E.G.D. Cohen, Nonlinearity 13, (2000) 1905-1924. 
[4] A. Pérez-Madrid, arXiv:cond-mat/0509491, to be published in J. Chem. Phys.

[5] N.N. Bogoliubov, Lectures on Quantum Statistics, Vol. 1, Gordon \& Breach, New York, 1967; J. von Neumann, Mathematical Foundations of Quantum Mechanics, Chap. IV, Princeton Univ. Press, Princeton, N. J., 1955.

[6] D.B. Boercker and J.W. Dufty, Annals of Phys. 119, (1979) 43-70.

[7] N.L. Balazs and B.K. Jennings, Phys. Reports 104, (1984) 347-391.

[8] M. Hillery, R.F. O'Connell, M.O. Scully and E.P. Wigner, Phys. Reports 106, (1984) 121-167.

[9] R. Balian, Y. Alhassid and H. Reinhardt, Phys. Reports 131, (1986) 1-146.

[10] J. Rau and B. Müller, Phys. Reports 272, (1996) 1-59.

[11] de Groot, S.R. and Mazur, P., 1984, Non-Equilibrium Thermodynamics (Dover, New York), Sect. VII.8, pag 126.

[12] van Kampen, N.G., 1990, Stochastic Processes in Physics and Chemistry (North-Holland, Amsterdam), Sect. VII.6, pag. 202.

[13] R. Balian, From Microphysics to Macrophysics, Vol. 1, Springer-Verlag, 1982; Sect. 3.2.2.

[14] E.H. Lieb, M.B. Ruskai, J. Math. Phys. 14, (1973) 1938-1941.

[15] G. Lindblad, Comm. Math. Phys. 40, (1975) 147-151.

[16] H. Spohn, J. Math. Phys. 19, (1978) 1227-1230.

[17] F. Schlögl, Phys. Rep. 62, (1980) 267-380.

[18] A. Wehrl, Rev. Modern Phys. 50, (1978) 221-260.

[19] R. Zwanzig, Lectures in Theoretical Physics (Boulder) (Interscience Publishers, Inc. New York, 1960), Vol.III.

[20] D. Bedeaux, P. Mazur, Physica A 298, (2001) 81-100 